\title{
Treatment planning comparison of volumetric modulated arc therapy employing a dual-layer stacked multi-leaf collimator and helical tomotherapy for cervix uteri
}

\author{
S. Panda ${ }^{1}$, J. Swamidas' ${ }^{1}$, S. Chopra ${ }^{1}$, A. Mangaj ${ }^{2}$, A. Fogliata ${ }^{3}$, P. Kupelian ${ }^{4,5}$, J. P. Agarwal ${ }^{2}$ and L. Cozzi ${ }^{3,6^{*}}$ (i)
}

\begin{abstract}
Purpose: To ascertain the dosimetric performance of a new delivery system (the Halcyon system, H) equipped with dual-layer stacked multi-leaf collimator (MLC) for risk-adapted targets in cervix uteri cancer patients compared to another ring-based system in clinical operation (Helical Tomotherapy, HT).

Methods: Twenty patients were retrospectively included in a treatment planning study (10 with positive lymph nodes and 10 without). The dose prescription (45Gy to the primary tumour volume and a simultaneously integrated boost up to 55Gy for the positive patients) and the clinical planning objectives were defined consistently as recommended by an ongoing multicentric clinical trial. Halcyon plans were optimised for the volumetric modulated arc therapy. The plan comparison was performed employing the quantitative analysis of the dose-volume histograms.

Results: The coverage of the primary and nodal target volumes was comparable for both techniques and both subsets of patients. The primary planning target volume (PTV) receiving at least $95 \%$ of the prescription isodose ranged from $97.2 \pm 1.1 \%$ (node-negative) to $99.1 \pm 1.2 \%$ (node-positive) for $\mathrm{H}$ and from $96.5 \pm 1.9 \%$ (node-negative) to $98.3 \pm$ $0.9 \%$ (node-positive) for HT. The uncertainty is expressed at one standard deviation from the cohort of patient per each group. For the nodal clinical target volumes, the dose received by $98 \%$ of the planning target volume ranged $55.5 \pm$ 0.1 to $56.0 \pm 0.8 \mathrm{~Gy}$ for $\mathrm{H}$ and $\mathrm{HT}$, respectively. The only significant and potentially relevant differences were observed for the bowels. In this case, $V_{40 G y}$ resulted $226.3 \pm 35.9$ and $186.9 \pm 115.9 \mathrm{~cm}^{3}$ for the node-positive and node-negative patients respectively for Halcyon. The corresponding findings for HT were: $258.9 \pm 60.5$ and $224.9 \pm 102.2 \mathrm{~cm}^{3}$. On the contrary, $\mathrm{V}_{15 \mathrm{~Gy}}$ resulted $1279.7 \pm 296.5$ and $1557.2 \pm 359.9 \mathrm{~cm}^{3}$ for $\mathrm{HT}$ and $\mathrm{H}$ respectively for node-positive and $1010.8 \pm 320.9$ versus $1203.8 \pm 332.8 \mathrm{~cm}^{3}$ for node-negative.

Conclusion: This retrospective treatment planning study, based on the dose constraints derived from the Embrace II study protocol, suggested the essential equivalence between Halcyon based and Helical Tomotherapy based plans for the intensity-modulated rotational treatment of cervix uteri cancer. Different levels of sparing were observed for the bowels with $\mathrm{H}$ better protecting in the high-dose region and $\mathrm{HT}$ in the mid-low dose regions. The clinical impact of these differences should be further addressed.
\end{abstract}

Keywords: Dual-layer MLC, Halcyon, Helical Tomotherapy, Cervix uteri, VMAT, RapidArc

\footnotetext{
* Correspondence: luca.cozzi@humanitas.it

${ }^{3}$ Humanitas Research Hospital, Radiotherapy and Cancer Center Radiosurgery

Dept, Via Manzoni 56, 20089 Milan-Rozzano, Italy

${ }^{6}$ Dept. of Biomedical Sciences, Humanitas University, Milan-Rozzano, Italy

Full list of author information is available at the end of the article
}

(c) The Author(s). 2020 Open Access This article is distributed under the terms of the Creative Commons Attribution 4.0 International License (http://creativecommons.org/licenses/by/4.0/), which permits unrestricted use, distribution, and reproduction in any medium, provided you give appropriate credit to the original author(s) and the source, provide a link to the Creative Commons license, and indicate if changes were made. The Creative Commons Public Domain Dedication waiver (http://creativecommons.org/publicdomain/zero/1.0/) applies to the data made available in this article, unless otherwise stated. 


\section{Introduction}

Primary chemoradiation and brachytherapy is the standard of care for patients with locally advanced cervical cancer. External beam radiation therapy (EBRT), brachytherapy, and concomitant chemotherapy are the integral parts of this treatment. However, these are associated with clinically significant acute and late toxicity. While most of the late sequelae in organs at risk (rectum, sigmoid and bladder) may be attributed to brachytherapy, a significant proportion of gastrointestinal acute and late sequelae are a result of both low and high doses received by the bowel. As the newer treatment algorithms envisage brachytherapy and nodal dose escalation, it's imperative that doses to organs at risk that potentially receive a contribution from both external radiation and brachytherapy are minimised to the lowest possible level. Also, in patients receiving extended field radiation due to para-aortic disease, higher incidence of gastrointestinal toxicity may be expected, and highly conformal radiation fields may help in reducing bowel dose. The use of Image-guided Intensity Modulated Radiation Therapy (IG-IMRT) is associated with reduced doses to the small bowel and may be associated with a reduction in patient-reported symptoms and physician-reported toxicity outcomes [1-5]. Similarly, IGIMRT for EBRT is associated with a reduced incidence of gastrointestinal toxicity.

Different variants of intensity-modulated radiotherapy (IMRT) techniques exist and, among these, the role of volumetric modulated arc therapy (VMAT) has been compared to fixed beam IMRT [6] confirming the potential of other rotational therapy techniques as Helical Tomotherapy (HT) [7-9].

After its clinical introduction in 2017, the performance of the new radiotherapy delivery platform Halcyon $(\mathrm{H}$, Varian Medical Systems, Palo Alto, USA) was investigated for a variety of different treatment indications [10-20]. In general, the potential performance equivalence between $\mathrm{H}$ and other carm delivery systems was proven for head and neck, breast, prostate [13] and brain tumours [15]. Some studies focused on cervix uteri cancer planning. With early versions of the Halcyon environment, Anamalayil [16], Brady [17] and Mihailidis [18] showed that: i) a good agreement was achievable between plans optimised for $\mathrm{H}$ or for other conventional platforms for both IMRT and VMAT and that ii) the use of multiple isocenters (with auto-feathering of overlapping fields/arcs) could overcome the field size limitations of $\mathrm{H}$. More recently, using the second generation release of the Halcyon system, Kim [19] reported about the clinical use of enhanced planning capabilities of $\mathrm{H}$ for the treatment of long targets using dual-isocenters. Li [20] compared the performance of Halcyon versus a conventional c-arm linac for IMRT.

The primary aim of the present study was to investigate the performance of Halcyon and Helical Tomotherapy for pelvic radiation for node-negative and node-positive patients according to the planning strategy of an ongoing multicentric international clinical trial, the Embrace II study [21], i.e. in a complex clinical strategy for gynaecological cancer. The protocol involves complex planning algorithm with specific dose constraints for various subvolumes for pelvic radiation. Furthermore, specific aims are also enlisted for nodal dose escalation while maintaining organ at risk doses.

The quality of the plans was compared for two rotational platforms. To further appraise the merit and potential issues of the new system, the Halcyon based plans were compared against the corresponding ones optimised for Helical Tomotherapy.

\section{Materials and methods}

Patient selection, target definition and dose prescription

A retrospective treatment planning study was performed on a cohort of 20 patients; 10 cases were selected with positive lymph nodes $(\mathrm{N}+)$ and 10 without positive nodes (N-) from the institutional trial database. Target delineation was performed according to the definitions and guidelines of the ongoing international clinical trial for gynaecological IG-IMRT [21]. In summary, target delineation was performed after fusing contrast-enhanced computed tomography $(\mathrm{CT})$ images with a resolution of $1.2 \times 1.2 \times 2.5 \mathrm{~mm}^{3}$ and $\mathrm{T} 2$ weighted magnetic resonance images (MRI, resolution of $5 \times 5 \times 3 \mathrm{~mm}^{3}$ ) registered to those images. The gross tumour volume (GTV) was delineated as the intermediate signal intensity region in the cervix and vagina on T2 MRI. The clinical target volumes (CTV) were delineated as follows: i) the high-risk CTV (CTV_HR, defined as the primary GTV and any remaining cervix not infiltrated by the tumour) and the low-risk CTV (CTV_LR) for the primary GTV including the parametria, the uterus and a margin of about $5 \mathrm{~mm}$ anterior and posterior towards bladder and rectum; ii) the elective CTV (CTV_E) for the elective nodal volume (including the nodal regions according to the risk stratification) and iii) the nodal CTV (CTV_N, with a further indication of the number of lymph-nodes (e.g. CTV N1)) for the lymph nodes (with a margin of $3 \mathrm{~mm}$ from the nodal GTV). The internal target volume (ITV) of the primary tumour (ITV45) was defined as the union of the CTV_E and the ITV from the CTV_LR (obtained adding a $10-\mathrm{mm}$ margin along the anterior-posterior and superior-inferior axes and $5 \mathrm{~mm}$ along the lateral axis), excluding muscles and bony boundaries in the pelvis. The planning target volumes (PTV45 and PTV_N respectively for the primary tumour and the nodal volumes) were generated with an isotropic margin of $5 \mathrm{~mm}$ from the corresponding ITVs or CTVs.

The dose prescription was set to $45 \mathrm{~Gy}$ in 25 fractions of 1.8Gy for PTV45. The nodal boost dose (for the $\mathrm{N}+$ subset) was optimised according to the simultaneous 
integrated boost (SIB) technique with a total dose of $55 \mathrm{~Gy} / 25$ fractions (2.2Gy per fraction). Dose optimisation and final computation were performed on contrast-free $\mathrm{CT}$ scans registered to the images used for segmentation.

The dose coverage to PTV45 was aimed to achieve that $95 \%$ of the volume is covered by the $95 \%$ isodose $\left(\mathrm{V}_{95 \%}>95\right)$. A stricter coverage was requested for the ITV45: $\mathrm{D}_{99 \%}>95$. For the planning target volumes of the pathological nodes, the required dose coverage was: $\mathrm{D}_{98 \%}>49.5 \mathrm{~Gy}$ (i.e. $90 \%$ of the prescription) while for the nodal clinical target volumes it was: $\mathrm{D}_{98 \%}>55 \mathrm{~Gy}(100 \%)$. The near-to-maximum dose objective was defined as $\mathrm{D}_{1 \%}<107 \%$.

The organs at risk (OAR) considered for the study were the bowel bag (defined as the outer contour of the loops including the mesenterium), the sigmoid, the bladder, the rectum, the spinal cord, the femoral heads and the kidneys. For each of these OARs, the specific dosevolume constraints were set for the near-to-maximum doses and for the organ-specific additional parameters. For the bladder, the rectum and the bowels, the volume objectives were enforced as soft constraints (due to the interpatient variability in the organs to target contouring), aiming to be achieved in about $70-80 \%$ of the cases. A detailed list of the constraints to the OARs is reported in the results together with the observed experimental findings.

\section{The treatment planning techniques and features}

Two sets of plans were designed and optimised for each patient in a full double-blind manner. Data analysis and comparison was performed only at the end of the planning phase, not allowing for further plan improvements. All plans were specifically designed and optimised for this in-silico treatment planning investigation.

Halcyon plans: The Halcyon is a ring-based delivery system [13, 22-24]. It consists of a linear accelerator capable of producing a $6 \mathrm{MV}$ flattening filter-free (FFF) photon beam delivering (when calibrated to deliver 1.0Gy per 100 monitor units (MU) at the reference conditions of source-surface distance (SSD) $100 \mathrm{~cm}$ at $\mathrm{d}_{\max }$ depth of $1.3 \mathrm{~cm}$ ) a maximum dose rate of $800 \mathrm{MU} / \mathrm{min}$. The linac rotates around the mounting ring with a velocity up to 4 rotations per minute in both imaging and delivery mode. The main features of Halcyon relevant for planning are: i) absence of the jaws in the $\mathrm{X}$ and $\mathrm{Y}$ directions; ii) a dual-layer stacked-staggered multi-leaf collimator; each layer is "shifted" $5 \mathrm{~mm}$ with respect to the other producing an effective shaping capability of 5 $\mathrm{mm}$ at isocenter. The design specifications of the multileaf collimator (MLC) stated a 0.01 transmission and leakage; iii) maximum field size for a single isocenter treatment of $28 \times 28 \mathrm{~cm}^{2}$ and $28.0 \mathrm{~cm}$ of overtravel of the MLC leaves with full inter-digitation; iv) possibility to automatically generate two isocenter plans for longer targets (up to $36.0 \mathrm{~cm}$ in Halcyon version 2) with autofeathering of overlapping fields/arcs.

All the plans were designed and optimised using the Eclipse treatment planning system (Varian Medical Systems, Palo Alto, USA) version 15.6. For each patient, VMAT plans were generated using a class solution consisting of 3 full arcs with collimator angles set to $10^{\circ}$, $350^{\circ}$ and $90^{\circ}$. A single isocenter was used for all $\mathrm{N}$ - cases and for $8 / 10 \mathrm{~N}+$ cases. In the remaining two cases, the target volume resulted longer than $28 \mathrm{~cm}$ and therefore the plans were optimised for two isocentres (with the same arcs arrangement) displaced of $8 \mathrm{~cm}$ in the $\mathrm{z}$ direction. The optimisation (and the delivery) is fully automated in Eclipse; field feathering is applied to accurately match the dose distribution in the overlapping region.

The VMAT optimisation in Eclipse was performed without applying constraints to the MLC complexity, i.e. leaving the system the freedom to adapt the "modulation intensity" case by case which is the planning default. For all plans, the dose distributions were computed using the Anisotropic Analytical Algorithm (AAA) [25] with a resolution of $2.5 \mathrm{~mm}$.

Helical Tomotherapy Plans: HT machine consists of a 6MV FFF beam with binary MLC, capable of a minimum leaf opening time of $20 \mathrm{~ms}$. The jaw widths are of size $1 \times 40,2.5 \times 40$ and $5 \times 40 \mathrm{~cm}^{2}$ at the Source to Axis distance of $85.0 \mathrm{~cm}$. The dose rate was $850 \mathrm{cGy} / \mathrm{min}$. For the current study, the field size was set to $5 \times 40 \mathrm{~cm}$ (with static jaws) and a pitch of 0.3 was used for all patients. The modulation factor was set to 3 for all plans. In HT, the delivery is helical in nature that enables long treatment lengths of up to $160 \mathrm{~cm}$, without any junction. Imaging is possible with a megavoltage fan-beam CT, for a maximum length of $160 \mathrm{~cm}$. The detailed machine characteristics can be found elsewhere [26-29]. All the plans were designed and optimised on the Hi-Art treatment planning system version 5.1.4 (Accuray, Sunnyvale, USA) with a resolution of $5 \mathrm{~mm}$ in the $x-y$ plane and 2.5 $\mathrm{mm}$ in $\mathrm{z}$.

Quantitative assessment: The analysis of the dose distributions aimed to quantify a variety of relevant metrics derived from the dose-volume histograms (DVH) for the various target volumes and OARs. For each structure, the mean dose and selected $V_{x}$ and $D_{x}$ parameters were extracted. $V_{x}$ represents the volume receiving at least an $x$ level of dose and $D_{x}$ the dose received by an $x$ fraction of volume. The metrics reported are relative to the dosevolume objectives suggested by the ongoing international clinical trial. For the planning target volumes, also the homogeneity index $\left(\operatorname{Homog}=\left(\mathrm{D}_{5 \%}-\mathrm{D}_{95 \%}\right) /\right.$ $\left.D_{\text {mean }}\right)$ was scored to measure the variance of the dose inside the volumes supposed to be homogeneously 
irradiated. Homogeneity was not part of the planning objectives set, but it was reported for consistency with literature standards. The conformity index (CI) was defined as the ratio of the volume receiving at least $36 \mathrm{~Gy}$ or $43 \mathrm{~Gy}$ to the volume of PTV45 $\left(\mathrm{CI}_{36 \mathrm{~Gy}}\right.$ and $\mathrm{Cl}_{43 \mathrm{~Gy}}$ respectively) and in terms of the Paddick conformity index ( $\left.\mathrm{CI}_{\text {Paddick }}\right)$ [30] computed for the 95\% isodose, i.e. 42.74Gy. The Paddick index was computed also for the sum of the SIB boost volumes (PTV_N1 and PTV_N2) for the 52.5Gy dose level (95\% of the boost dose prescription) and reported as $\mathrm{CI}_{\text {Paddick_SIB }}$. To complete the analysis, also the average DVH were computed, for each structure and each cohort with a dose binning resolution of $0.02 \mathrm{~Gy}$. All dose-volume metrics were derived from the systems the plans were optimised in. In-house tools were used to generate the average DVH for the images. All dose distributions were imported in the Eclipse system for the graphical comparison. For both techniques the estimated treatment time (excluding imaging) was derived from the plans and reported as a further comparative factor.
The assessment of the potential statistical significance of the differences among the groups of plans was performed using non-parametric tests (Wilcoxon test for paired samples).

\section{Results}

Figures 1 and 2 present the isodose distributions in colour-wash for two example patients from the $\mathrm{N}+$ and $\mathrm{N}$ - cohorts. The qualitative assessment demonstrates very similar degrees of conformality and sparing of organs at risk for both $\mathrm{H}$ and $\mathrm{HT}$ plans.

Figures 3 and 4 show the average dose-volume histograms computed for the two subgroups of patients. Data are shown for the various target volumes and the organs at risk. The quantitative analysis of the DVHs is summarised in Table 1 (for the target volumes) and Tables 23 for the OARs.

Starting from the $\mathrm{N}+$ cases, the required dose coverage measured by $V_{95 \%}$ for PTV45, $D_{98 \%}$ for CTV_N and PTV_N and $\mathrm{D}_{99 \%}$ (near-to-minimum dose) for ITV45, was achieved for both $\mathrm{H}$ and HT plans. Data shows a systematic, although modest, improvement for $\mathrm{H}$ over

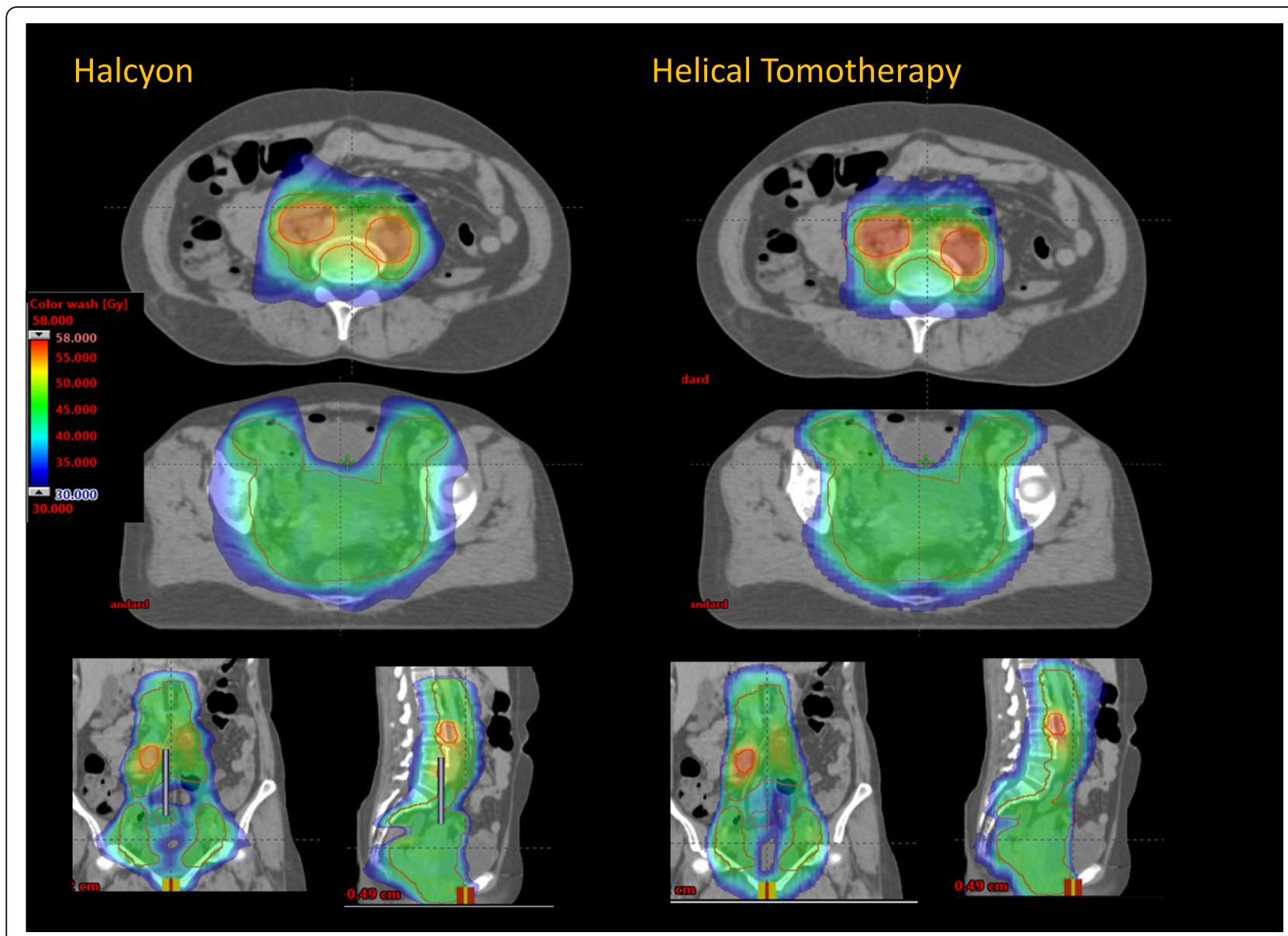

Fig. 1 Isodose distributions in colour wash (scale between 35 and 48Gy) for one example of node-positive patient in two axial, coronal and sagittal planes 


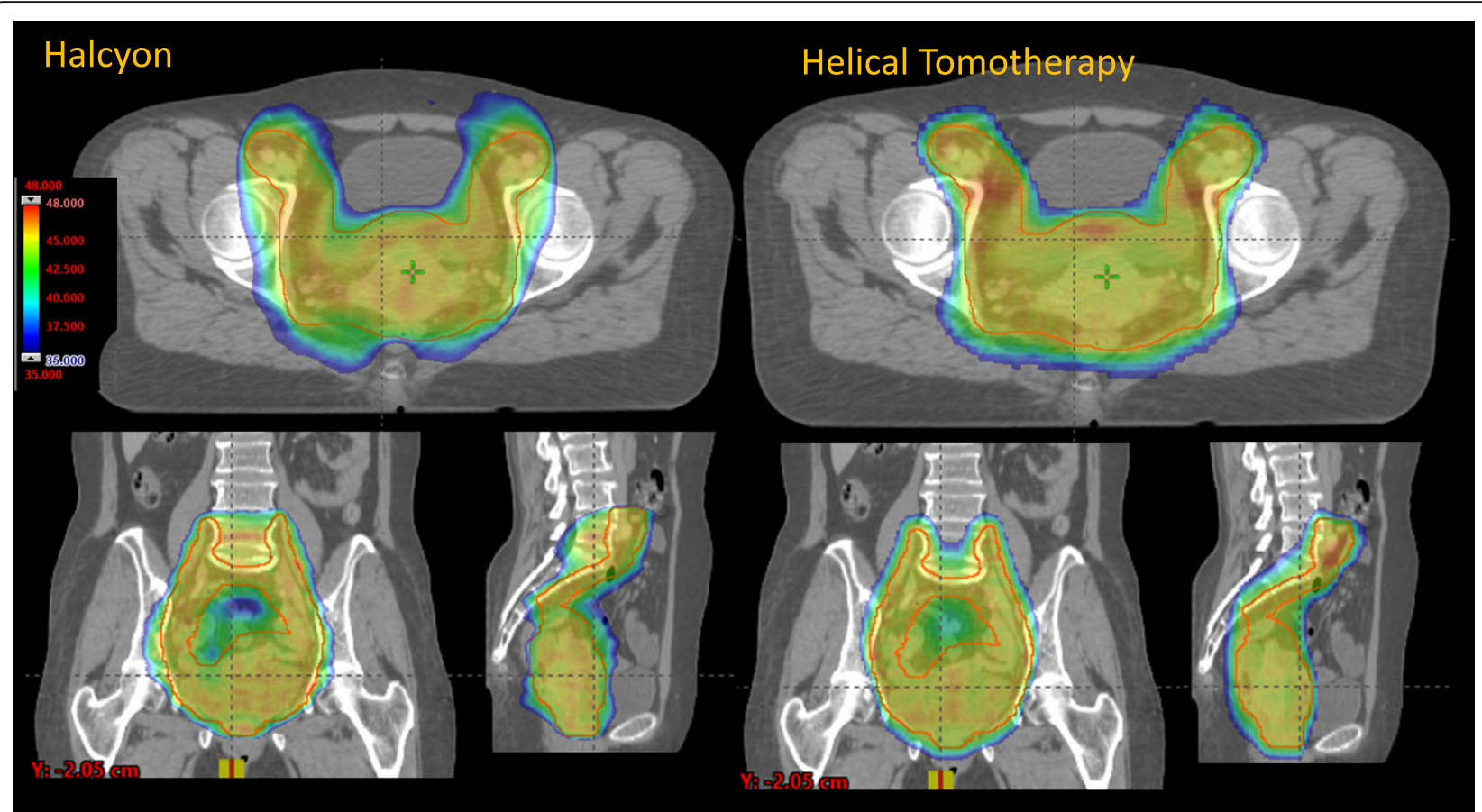

Fig. 2 Isodose distribution in colour wash (scale between 35 and 48Gy) for one example of node-negative patients in an axial, coronal and sagittal planes

$\mathrm{HT}$ as measured by the positive difference between $\mathrm{H}$ and HT results. The results for $\mathrm{H}$ demonstrated also a modest reduction (smaller than 1Gy for PTV45 and than 2Gy for the PTV_N) of the near-to-maximum doses, reported by $D_{1 \%}$. The findings from coverage and maximum dose reflected in the improved homogeneity of $\mathrm{H}$ versus HT; to note that homogeneity was not part of the optimisation criteria. The median dose to the ITV45 resulted in average coherent with the aimed 45Gy with a modest but significant over-dosage observed in the HT data (0.7Gy higher than the $\mathrm{H}$ plans). The median dose for the nodal CTV (CTV_N1 and CTV_N2) resulted on average slightly inferior to the objective of 55Gy for $\mathrm{H}$ and about 2Gy higher for HT. In summary, the use of the SIB technique for the $\mathrm{N}+$ cases did not impact in any significant manner on the quality of the plans in the comparison between the two delivery systems.

Concerning the simpler case of the N-, the PTV45 or ITV45 coverage (expressed by $\mathrm{V}_{95 \%}$ and $\mathrm{D}_{99 \%}$ respectively) was met by both techniques with $\mathrm{H}$ data improving the HT findings of $0.7 \%$ (not significant) or 3.3\% respectively. In this case, the homogeneity of the $\mathrm{H}$ plans resulted about $1 \%$ worse than $\mathrm{HT}$, in an opposite manner to what reported for the $\mathrm{N}+$ cases. The median dose to the ITV45 resulted consistent with the constraint of 45Gy for both $\mathrm{H}$ and $\mathrm{HT}$ without significant differences.

Although most of the differences between $\mathrm{H}$ and HT plans resulted statistically significant, their modest absolute value might not impact at the clinical level.
Concerning the OARs, the results are more variable. For the sigmoid, the spinal cord, the femoral heads and the kidneys both delivery systems mostly met the planning objectives. For the femoral heads, although not subject to any constraints in the optimisation, the HT plans better spared the entire structures resulting in significantly lower mean doses. In most of the cases, no statistical significance was measured in the comparison of the groups. The optimisation of the dose distribution for the bowels, the bladder and the rectum resulted more challenging.

For the bowels, the EMBRACE II required as a hard constraint the maximum dose to be lower than 47.3 or $57.5 \mathrm{~Gy}$ for the $\mathrm{N}$ - and $\mathrm{N}+$ plans respectively. Both delivery systems achieved this request for all patients in terms of the near-to-maximum parameter.

Soft objectives were enforced to spare the volume receiving 30 or 40 Gy below 500 and $250 \mathrm{~cm}^{3}$ for the $\mathrm{N}+$ patients ( 350 and $100 \mathrm{~cm}^{3}$ respectively for the $\mathrm{N}-$ ). For $\mathrm{N}+$ patients, $\mathrm{H}$ resulted in a significant additional sparing of in average 60 and $33 \mathrm{~cm}^{3}$ for $\mathrm{N}+$ and 62 and $38 \mathrm{~cm}^{3}$ for the N-patients. HT plans presented a lower dose spillage $\left(\mathrm{V}_{15 \mathrm{~Gy}}\right)$ which, although a parameter not included in the optimisation constraints, is a desirable result: $\mathrm{V}_{15 \mathrm{~Gy}}$ resulted $1279.7 \pm 296.5$ and $1557.2 \pm 359.9 \mathrm{~cm}^{3}$ for HT and $\mathrm{H}$ respectively for node-positive and $1010.8 \pm 320.9$ versus $1203.8 \pm 332.8 \mathrm{~cm}^{3}$. The planning objectives were on average met for $\mathrm{H}$ in the $\mathrm{N}+$ cohort and modestly violated (for $\mathrm{V}_{\text {30Gy }}$ ) for HT. The objectives were, on average, not met for the $\mathrm{N}$ - patients. The considerable uncertainty (at one 


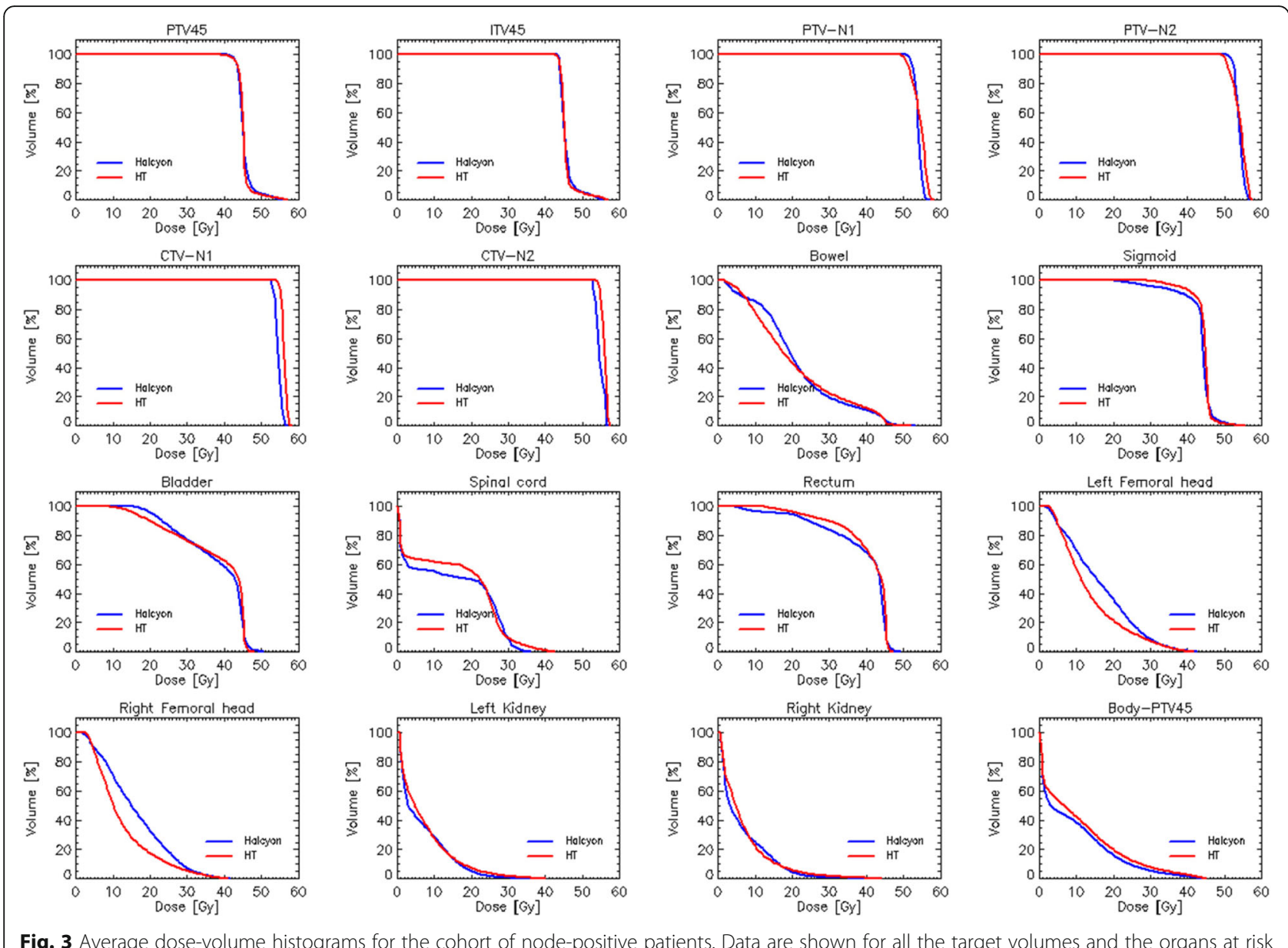

standard deviation) is due to the large inter-patient variability in the contouring and in the size of the bowel bags; of course, the interpatient variability in contouring arises from the difference in tumour volumes and ITV generation that represents the real-life situation. Concerning $\mathrm{N}+$ patients, the $\mathrm{V}_{40 \text { Gy }}$ objective was respected in $7 / 10$ cased for $\mathrm{H}$ and in $4 / 10$ for HT. $\mathrm{V}_{30 \text { Gy }}$ in 10/10 cases for $\mathrm{H}$ and in 7/10 for HT. Concerning N- patients, $\mathrm{V}_{40 \text { Gy }}$ was achieved in 2 patients while $V_{30 G y}$ in 6 patients for the $H$ plans. In the $\mathrm{HT}$ case, no patient respected the $\mathrm{V}_{40 \mathrm{~Gy}} \mathrm{ob}-$ jective and only four the $V_{30 G y}$. As an additional metric, not explicitly enforced in the optimisation process, $V_{15 G y}$ resulted significantly better for $\mathrm{HT}$ plans in both $\mathrm{N}+$ and $\mathrm{N}$ - subsets.

Concerning the Rectum, on average the soft constraints were met for both $\mathrm{H}$ and $\mathrm{HT}$ in the $\mathrm{N}+$ set and not met by HT for the $\mathrm{N}$ - cases. Halcyon plans allowed some further (and statistically significant for the $\mathrm{N}$ cases) sparing of the rectum compared to the Helical tomotherapy plans. The considerable uncertainty associated with the mean values is primarily due to the interpatient variability in the position of the rectum with respect to the target volumes. A similar trend, although very modest and not significant, was also observed for the bladder.

The Halcyon plans resulted more conformal than the HT as demonstrated by both the conformality indices with an average gain in the index ranging from 0.1 to 0.4. The $\mathrm{CI}_{\text {Paddick }}$ for the PTV45 resulted better for HT in the $\mathrm{N}+$ cases (and this was also for the Paddick conformity index computed on the nodal volumes) and slightly better for $\mathrm{H}$ in the $\mathrm{N}$ - cases.

The estimated treatment time for the HT plans was $3.6 \pm 0.3 \mathrm{~min}$ and $5.2 \pm 0.5 \mathrm{~min}$ for the $\mathrm{N}$ - and $\mathrm{N}+$ cases respectively. The estimated treatment time for the $\mathrm{H}$ plans was $2.4 \pm 0.3$ and $2.9 \pm 0.2$ respectively (inclusive of collimator rotation and multiple isocenter setting for the two $\mathrm{N}+$ cases requiring it) since with conventional fractionation the delivery time is mainly given by the rotational velocity of the system which is typically 2 rotations per minute (possibly increasing to 3 ).

\section{Discussion}

The main scope of this treatment planning investigation was to verify the hypothesis that the new delivery platform Halcyon was adequate to achieve plan quality 

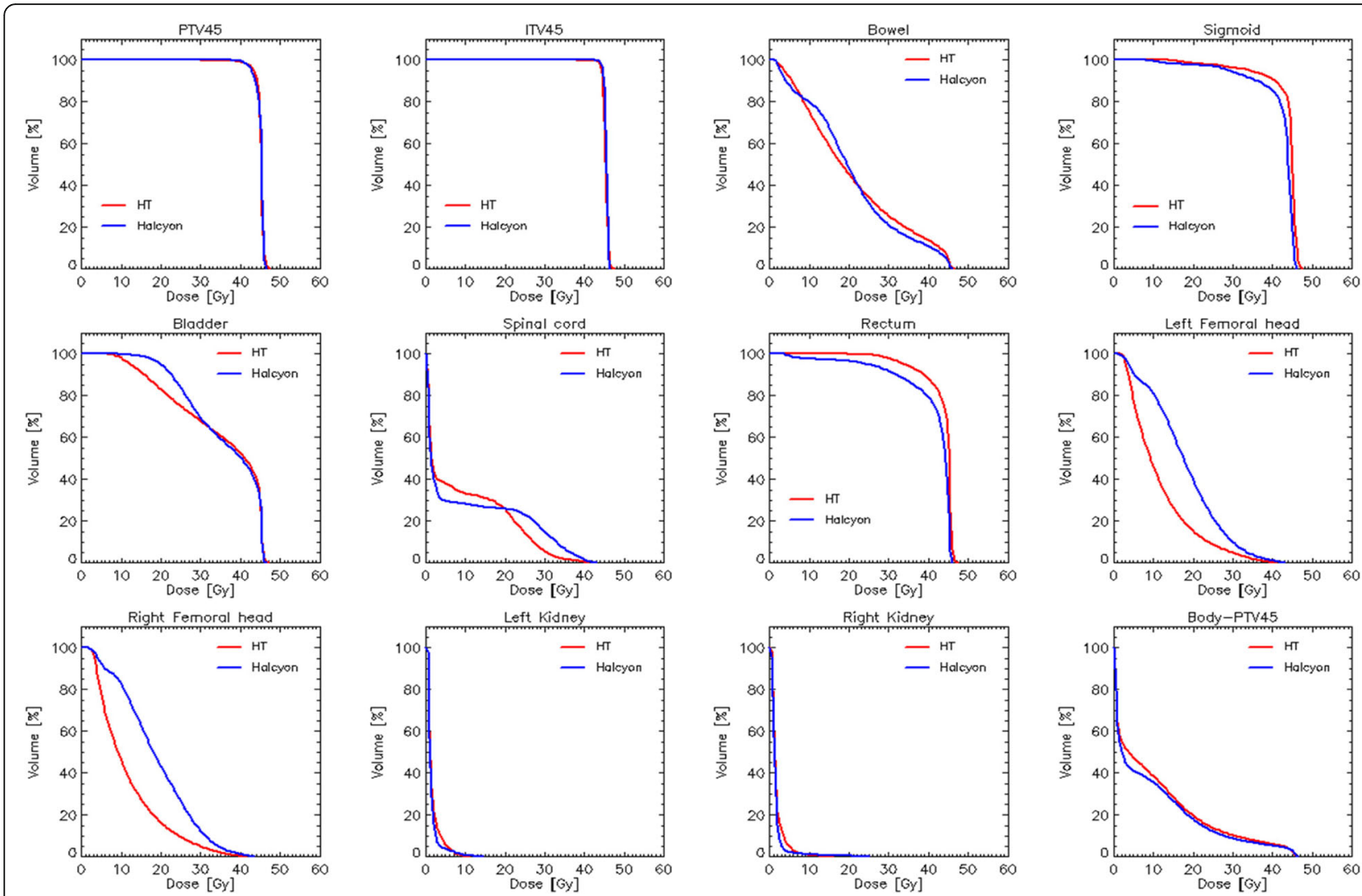

Fig. 4 Average dose volume histograms for the cohort of node-negative patients. Data are shown for all the target volumes and the organs at risk

consistent (equivalent or better) to other consolidated advanced practices and to fulfil stringent clinical protocols. The unique features of Halcyon (mainly the double layer MLC) justify the need for pre-clinical dosimetric investigations to define the field of applicability or the deficiencies of the platform. As a comparison technique, as a novelty factor compared to [13], the Helical Tomotherapy was chosen. The role of HT for cervix uteri was appraised in several studies showing how complex dose distributions with a high degree of conformal avoidance (simultaneous achievement of target coverage and healthy tissue sparing) could be achieved [7-9].

The data summarised in this report confirmed the hypothesis. Halcyon based plans resulted substantially equivalent to Helical Tomotherapy in terms of target volume coverage and allowed for an equivalent or improved sparing of the various OARs. In particular, the sparing of the bowels resulted in about 15-20\% further saving (on average ranging from 30 to $60 \mathrm{~cm}^{3}$ for $\mathrm{V}_{40 \mathrm{~Gy}}$ and $\mathrm{V}_{30 \mathrm{~Gy}}$. HT presented, on the contrary, a lower spillage, e.g. measured by $\mathrm{V}_{15 G y}$, a predictor of diahrrea related toxicity. This fact allowed to better meet the challenging clinical aims for the bowel (particularly for the $\mathrm{N}$ - subset). The quantification of the associated reduction of toxicity should follow proper clinical validation, although it might be hardly detectable with such a modest dose difference. From a speculative point of view, since both parameters are predictors of toxicity, Halcyon plans should be considered advantageous. All other differences in the organs at risk resulted either marginally or not statistically significant, proving the equivalence of $\mathrm{H}$ and $\mathrm{HT}$ for the remaining metrics.

Kim [19] reported about the treatment of 12 patients treated with extended-field intensity-modulated radiotherapy planned with the dual isocenter technique applied also in the present study for long targets. The dose prescription was equivalent to the Embrace regimen for the $\mathrm{N}+$ patients while the dose-volume objectives for the OARs were different but similarly demanding. Authors demonstrated the capability of Halcyon to generate and deliver the needed high-quality plans for all cases using the dual isocenter approach. The pretreatment dosimetric verification of the plans with a $2 \mathrm{D}$ array of ion chambers resulted ranging from 97.3 to $99.9 \%$ (with $3 \% / 3 \mathrm{~mm}$ thresholds in the distance to agreement and dose difference parameters). The total treatment time was reported in the range of 5 to $6.5 \mathrm{~min}$ per fraction.

The potential of Halcyon for cervix uteri was investigated also by $\mathrm{Li}$ [20], over a group of 30 patients with a dose prescription of 50.4Gy and no SIB and with dose-volume aims 
Table 1 Summary of the dose volume histogram analysis for the target volumes. Data are presented for the node positive and node negative patients

\begin{tabular}{|c|c|c|c|c|c|}
\hline Parameter & Objective & Halcyon $(\mathrm{H})$ & Helical Tomotherapy (HT) & Difference $(\mathrm{H}-\mathrm{HT})$ & $\mathrm{p}$ \\
\hline \multicolumn{6}{|l|}{ Node Positive } \\
\hline \multicolumn{6}{|l|}{ PTV45 } \\
\hline$V_{95 \%}[\%]$ & $\geq 95$ & $99.1 \pm 1.2$ & $98.3 \pm 0.9$ & $0.8 \pm 0.9$ & 0.02 \\
\hline $\mathrm{D}_{1 \%}[\%]$ & $\leq 130^{b}$ & $122.9 \pm 1.7$ & $123.2 \pm 3.8$ & $-0.3 \pm 2.8$ & 0.73 \\
\hline Homog [\%] & a & $2.8 \pm 3.1$ & $3.3 \pm 2.1$ & $-0.5 \pm 2.6$ & 0.002 \\
\hline \multicolumn{6}{|l|}{ ITV45 } \\
\hline$D_{99 \%}[\%]$ & $\geq 95$ & $100.1 \pm 1.2$ & $97.6 \pm 1.3$ & $2.5 \pm 1.1$ & $<0.001$ \\
\hline $\mathrm{D}_{50 \%}[\mathrm{~Gy}]$ & 45.0 & $45.0 \pm 0.4$ & $45.8 \pm 0.5$ & $-0.7 \pm 0.6$ & 0.002 \\
\hline \multicolumn{6}{|l|}{ PTV_N1 } \\
\hline $\mathrm{D}_{1 \%}[\mathrm{~Gy}]$ & $\leq 58.9$ & $56.2 \pm 0.2$ & $58.0 \pm 0.6$ & $-1.8 \pm 0.6$ & $<0.01$ \\
\hline $\mathrm{D}_{98 \%}[\mathrm{~Gy}]$ & $\geq 49.5$ & $53.8 \pm 0.3$ & $51.2 \pm 1.2$ & $2.6 \pm 1.1$ & $<0.01$ \\
\hline Homog [\%] & a & $3.8 \pm 2.7$ & $8.9 \pm 3.5$ & $-5.1 \pm 3.3$ & $<0.001$ \\
\hline \multicolumn{6}{|l|}{ PTV_N2 } \\
\hline $\mathrm{D}_{1 \%}[\mathrm{~Gy}]$ & $\leq 58.9$ & $56.3 \pm 0.2$ & $58.1 \pm 0.7$ & $-1.2 \pm 1.0$ & $<0.001$ \\
\hline$D_{98 \%}[G y]$ & $\geq 49.5$ & $53.6 \pm 0.3$ & $51.0 \pm 1.2$ & $1.8 \pm 1.5$ & $<0.001$ \\
\hline Homog [\%] & a & $3.9 \pm 3.1$ & $9.1 \pm 4.0$ & $5.2 \pm 4.0$ & $<0.001$ \\
\hline \multicolumn{6}{|l|}{ CTV_N1 } \\
\hline $\mathrm{D}_{98 \%}[\mathrm{~Gy}]$ & $\geq 55.0$ & $55.5 \pm 0.1$ & $56.0 \pm 0.8$ & $-0.5 \pm 0.9$ & 0.10 \\
\hline $\mathrm{D}_{50 \%}[\mathrm{~Gy}]$ & 55.0 & $54.5 \pm 0.9$ & $57.1 \pm 0.5$ & $-2.5 \pm 1.2$ & $<0.001$ \\
\hline \multicolumn{6}{|l|}{ CTV_N2 } \\
\hline$D_{98 \%}[G y]$ & $\geq 55.0$ & $55.5 \pm 0.2$ & $55.5 \pm 0.4$ & $-0.1 \pm 0.3$ & 0.63 \\
\hline$D_{50 \%}[G y]$ & 55.0 & $54.4 \pm 1.1$ & $57.1 \pm 0.5$ & $-2.7 \pm 1.2$ & $<0.001$ \\
\hline \multicolumn{6}{|l|}{ Node Negative } \\
\hline \multicolumn{6}{|l|}{ PTV45 } \\
\hline$V_{95 \%}[\%]$ & $\geq 95$ & $97.2 \pm 1.1$ & $96.5 \pm 1.9$ & $0.7 \pm 1.9$ & 0.27 \\
\hline $\mathrm{D}_{1 \%}[\%]$ & $\leq 107$ & $104.2 \pm 0.9$ & $103.1 \pm 0.7$ & $0.1 \pm 0.1$ & 0.003 \\
\hline Homog [\%] & a & $4.5 \pm 1.5$ & $3.2 \pm 2.1$ & $1.3 \pm 0.9$ & 0.007 \\
\hline \multicolumn{6}{|l|}{ ITV45 } \\
\hline $\mathrm{D}_{99 \%}[\%]$ & $\geq 95$ & $100.0 \pm 0.8$ & $96.5 \pm 1.5$ & 3.3 & $<0.001$ \\
\hline $\mathrm{D}_{50 \%}[\mathrm{~Gy}]$ & 45.0 & $45.4 \pm 0.1$ & $45.1 \pm 0.2$ & $0.3 \pm 2$ & 0.23 \\
\hline
\end{tabular}

a: not included as optimisation constraint

${ }^{b}$ : due to the simultaneous irradiation of the boost volumes. $V_{x} \%$ volume receiving at least $x \%$ of the dose. $D_{x \%}$ : dose received by at least $x \%$ of the volume. Homog $=\left(\mathrm{D} 5-\mathrm{D}_{95}\right) / \mathrm{D}_{\text {mean }}$

different from the highly demanding Embrace constraints, i.e. in a substantially different setting from what applied in the present investigation. For IMRT plans, authors demonstrated equivalence between Halcyon and c-arm linac based plans for the target volume while achieved improved sparing of organs at risk with $\mathrm{H}$. More importantly, Li reported about the pre-treatment dosimetric verifications of plans. The verification of $\mathrm{H}$ plans resulted in an average gamma index passing rate of $99.41 \pm 0.26$ using $3 \mathrm{~mm} / 3 \%$ thresholds in the distance to agreement and dose difference parameters.

De Roover [31] performed validation of intensitymodulated and VMAT plans according to the American Association of Physicists in Medicine task group 119
[32] on some test cases, on anthropomorphic phantoms and on the first patients treated in their clinic. Authors reported gamma agreement scores ranging from 97.0 to $100.0 \%$ for the test cases (film dosimetry) and above $95.8 \%$ for the patients (diode dosimetry).

The pretreatment verification was out of the scope of our current investigation since HT is a consolidated platform while for $\mathrm{H}$, we assume the validity of the literature data, specific for cervix uteri, reviewed above.

Compared to these two recent investigations, our study focused on the comparative assessment of the potential of Halcyon versus a consolidated technique from the Tomotherapy platform in the frame of the planning 
Table 2 Summary of the dose volume histogram analysis for the organs at risk for the node positive patients

\begin{tabular}{|c|c|c|c|c|c|}
\hline Parameter & Objective & Halcyon $(\mathrm{H})$ & Helical Tomotherapy (HT) & Difference $(\mathrm{H}-\mathrm{HT})$ & $p$ \\
\hline \multicolumn{6}{|l|}{ Bowel } \\
\hline Mean [Gy] & Minimise & $21.2 \pm 3.1$ & $21.1 \pm 2.8$ & $0.1 \pm 0.9$ & 0.82 \\
\hline $\mathrm{D}_{1 \%}[\mathrm{~Gy}]$ & $\leq 57.5$ & $48.8 \pm 1.7$ & $47.4 \pm 1.3$ & $1.4 \pm 0.7$ & $<0.001$ \\
\hline$V_{40 G y}\left[\mathrm{~cm}^{3}\right]$ & $\leq 250^{\mathrm{a}}$ & $226.3 \pm 35.9$ & $258.9 \pm 60.5$ & $-32.6 \pm 34.3$ & 0.01 \\
\hline$V_{30 G y}\left[\mathrm{~cm}^{3}\right]$ & $\leq 500^{a}$ & $413.4 \pm 58.9$ & $472.7 \pm 90.0$ & $-59.4 \pm 53.5$ & 0.01 \\
\hline$V_{15 \mathrm{~Gy}}\left[\mathrm{~cm}^{3}\right]$ & $\leq 700^{b}$ & $1557.2 \pm 359.9$ & $1279.7 \pm 296.5$ & $277.4 \pm 188.6$ & 0.01 \\
\hline \multicolumn{6}{|l|}{ Sigmoid } \\
\hline$D_{1 \%}[G y]$ & $\leq 57.5$ & $50.5 \pm 3.7$ & $49.4 \pm 3.8$ & $1.1 \pm 2.1$ & 0.11 \\
\hline \multicolumn{6}{|l|}{ Bladder } \\
\hline$D_{1 \%}[G y]$ & $\leq 57.5$ & $48.8 \pm 2.2$ & $47.0 \pm 1.1$ & $1.8 \pm 1.8$ & 0.01 \\
\hline $\mathrm{V}_{40 G y}[\%]$ & $\leq 75^{a}$ & $60.0 \pm 10.8$ & $63.6 \pm 16.6$ & $-3.6 \pm 7.5$ & 0.16 \\
\hline$V_{30 G y}[\%]$ & $\leq 85^{a}$ & $76.9 \pm 7.1$ & $77.0 \pm 12.5$ & $-0.1 \pm 9.2$ & 0.96 \\
\hline \multicolumn{6}{|l|}{ Rectum } \\
\hline $\mathrm{D}_{1 \%}[\mathrm{~Gy}]$ & $\leq 57.5$ & $48.1 \pm 1.3$ & $46.9 \pm 0.7$ & $1.2 \pm 1.8$ & 0.05 \\
\hline $\mathrm{V}_{40 G y}[\%]$ & $\leq 85^{a}$ & $66.6 \pm 20.5$ & $72.8 \pm 21.7$ & $-6.2 \pm 11.2$ & 0.11 \\
\hline$V_{30 G y}[\%]$ & $\leq 95^{a}$ & $81.9 \pm 12.7$ & $89.7 \pm 14.7$ & $-7.8 \pm 12.2$ & 0.07 \\
\hline \multicolumn{6}{|l|}{ Spinal Cord } \\
\hline$D_{1 \%}[G y]$ & $\leq 48.0$ & $25.1 \pm 17.9$ & $25.6 \pm 16.5$ & $-2.4 \pm 4.4$ & 0.12 \\
\hline \multicolumn{6}{|l|}{ Left Fem. Head } \\
\hline $\mathrm{D}_{1 \%}[\mathrm{~Gy}]$ & $\leq 50.0$ & $38.1 \pm 3.6$ & $39.4 \pm 1.6$ & $-1.3 \pm 3.2$ & 0.24 \\
\hline Mean [Gy] & b & $16.4 \pm 2.7$ & $13.8 \pm 2.5$ & $2.6 \pm 3.1$ & 0.001 \\
\hline \multicolumn{6}{|c|}{ Right Fem. Head } \\
\hline $\mathrm{D}_{1 \%}[\mathrm{~Gy}]$ & $\leq 50.0$ & $36.3 \pm 4.6$ & $41.7 \pm 2.9$ & $-5.4 \pm 2.5$ & $<0.001$ \\
\hline Mean [Gy] & b & $16.1 \pm 2.3$ & $12.6 \pm 1.3$ & $3.5 \pm 1.9$ & $<0.001$ \\
\hline \multicolumn{6}{|l|}{ Left Kidney } \\
\hline Mean [Gy] & $\leq 15$ & $6.9 \pm 5.0$ & $7.5 \pm 5.1$ & $-0.6 \pm 1.5$ & 0.22 \\
\hline \multicolumn{6}{|l|}{ Right Kidney } \\
\hline Mean [Gy] & $\leq$ & $6.6 \pm 4.8$ & $7.1 \pm 4.2$ & $-0.5 \pm 2.1$ & 0.52 \\
\hline \multicolumn{6}{|l|}{ Body } \\
\hline$D_{1 \%}[G y]$ & $\leq 58.9$ & $53.2 \pm 1.4$ & $49.9 \pm 3.7$ & $3.3 \pm 4.1$ & $<0.001$ \\
\hline $\mathrm{Cl}_{36 \mathrm{~Gy}}$ & $<1.6^{\mathrm{b}}$ & $1.6 \pm 0.2$ & $2.0 \pm 0.1$ & $-0.4 \pm 0.2$ & $<0.001$ \\
\hline $\mathrm{Cl}_{43 \mathrm{~Gy}}$ & $<1.1^{\mathrm{b}}$ & $1.1 \pm 0.1$ & $1.3 \pm 0.1$ & $-0.2 \pm 0.1$ & $<0.001$ \\
\hline $\mathrm{Cl}_{\text {Paddick }}$ & b & $0.87 \pm 0.04$ & $0.90 \pm 0.07$ & $-0.03 \pm 0.08$ & 0.01 \\
\hline $\mathrm{Cl}_{\text {Paddick_SIB }}$ & b & $0.71 \pm 0.08$ & $0.78 \pm 0.15$ & $-0.07 \pm 0.10$ & 0.05 \\
\hline
\end{tabular}

$\mathrm{V}_{\mathrm{x} \%}$ : volume receiving at least $\mathrm{x} \%$ of the dose. $\mathrm{D}_{\mathrm{x} \%}$ : dose received by at least $\mathrm{x} \%$ of the volume

a: soft constraints, expected to be fulfilled in $70-80 \%$ of the patients according to EMBRACE II guidelines

b: not included as optimisation constraint

rules set by a demanding clinical protocol. All three independent investigations confirm the capability of $\mathrm{H}$ to perform at a high level for this challenging clinical indication.

By design, the present study was conducted in doubleblind; the results presented are therefore not biased toward one technique. The fundamental impact of the planner's skills on each technique was mitigated by selecting highly experienced operators for the optimisation of each set of plans.

It is therefore essential to outline the fact that the equivalence/modest superiority of Halcyon was achieved strictly adopting the same clinical dose-volume aims as in an international protocol converted into optimisation constraints tailored to the optimisation engines applied for Halcyon or Helical Tomotherapy. 
Table 3 Summary of the dose volume histogram analysis for the organs at risk for the node negative patients

\begin{tabular}{|c|c|c|c|c|c|}
\hline Parameter & Objective & Halcyon $(\mathrm{H})$ & Helical Tomotherapy (HT) & Difference $(\mathrm{H}-\mathrm{HT})$ & $\mathrm{p}$ \\
\hline \multicolumn{6}{|l|}{ Bowel } \\
\hline Mean [Gy] & Minimise & $20.5 \pm 4.0$ & $21.0 \pm 3.0$ & $-0.6 \pm 1.7$ & 0.7 \\
\hline$V_{40 G y}\left[\mathrm{~cm}^{3}\right]$ & $\leq 100^{a}$ & $186.9 \pm 115.9$ & $224.9 \pm 102.2$ & $-38.1 \pm 22.2$ & $<0.001$ \\
\hline$V_{30 G y}\left[\mathrm{~cm}^{3}\right]$ & $\leq 350^{a}$ & $360.9 \pm 195.0$ & $422.9 \pm 134.2$ & $-62.1 \pm 72.1$ & 0.02 \\
\hline$V_{15 \mathrm{~Gy}}\left[\mathrm{~cm}^{3}\right]$ & $\leq 600^{b}$ & $1203.8 \pm 332.9$ & $1010.8 \pm 320.9$ & $192.9 \pm 160.4$ & 0.4 \\
\hline \multicolumn{6}{|l|}{ Sigmoid } \\
\hline$D_{1 \%}[G y]$ & $\leq 47.3$ & $46.1 \pm 0.5$ & $46.1 \pm 0.8$ & $-0.08 \pm 0.89$ & 0.78 \\
\hline \multicolumn{6}{|l|}{ Bladder } \\
\hline$D_{1 \%}[G y]$ & $\leq 47.3$ & $46.6 \pm 0.2$ & $46.0 \pm 0.3$ & $0.58 \pm 0.28$ & $<0.001$ \\
\hline$V_{40 G y}[\%]$ & $\leq 75^{a}$ & $50.4 \pm 7.0$ & $52.4 \pm 8.3$ & $-2.1 \pm 6.6$ & 0.35 \\
\hline$V_{30 G y}[\%]$ & $\leq 85^{a}$ & $69.6 \pm 5.8$ & $67.6 \pm 7.2$ & $2.0 \pm 8.6$ & 0.47 \\
\hline \multicolumn{6}{|l|}{ Rectum } \\
\hline $\mathrm{D}_{1 \%}[\mathrm{~Gy}]$ & $\leq 47.3$ & $46.2 \pm 0.4$ & $46.1 \pm 0.6$ & $0.10 \pm 0.89$ & 0.72 \\
\hline$V_{40 G y}[\%]$ & $\leq 85^{a}$ & $80.8 \pm 10.8$ & $88.1 \pm 12.3$ & $-7.2 \pm 11.6$ & 0.08 \\
\hline$V_{30 G y}[\%]$ & $\leq 95^{a}$ & $92.4 \pm 6.4$ & $97.4 \pm 3.9$ & $-5.0 \pm 6.1$ & 0.03 \\
\hline \multicolumn{6}{|c|}{ Left Fem. Head } \\
\hline $\mathrm{D}_{1 \%}[\mathrm{~Gy}]$ & $\leq 50.0$ & $38.7 \pm 6.9$ & $32.6 \pm 11.7$ & $6.1 \pm 12.7$ & 0.17 \\
\hline Mean [Gy] & b & $18.1 \pm 2.7$ & $11.7 \pm 0.9$ & $6.3 \pm 2.2$ & $<0.001$ \\
\hline \multicolumn{6}{|c|}{ Right Fem. Head } \\
\hline$D_{1 \%}[G y]$ & $\leq 50.0$ & $38.5 \pm 4.4$ & $36.5 \pm 3.6$ & $1.9 \pm 4.4$ & 0.20 \\
\hline Mean [Gy] & b & $18.8 \pm 3 . .3$ & $11.7 \pm 0.9$ & $6.9 \pm 2.7$ & $<0.001$ \\
\hline \multicolumn{6}{|l|}{ Left Kidney } \\
\hline Mean [Gy] & $\leq 15$ & $1.5 \pm 0.7$ & $1.5 \pm 0.8$ & $-0.01 \pm 0.32$ & 0.97 \\
\hline \multicolumn{6}{|l|}{ Right Kidney } \\
\hline Mean [Gy] & $\leq 15$ & $1.5 \pm 1.0$ & $1.6 \pm 0.9$ & $-0.11 \pm 3.7$ & 0.37 \\
\hline \multicolumn{6}{|l|}{ Body } \\
\hline$D_{1 \%}[G y]$ & $\leq 47.3$ & $41.9 \pm 1.1$ & $42.5 \pm$ & $0.85 \pm 0.59$ & 0.001 \\
\hline $\mathrm{Cl}_{36 \mathrm{~Gy}}$ & $<1.6^{b}$ & $1.56 \pm 0.08$ & $1.82 \pm 0.19$ & $-0.26 \pm 0.16$ & 0.19 \\
\hline $\mathrm{Cl}_{43 G y}$ & $<1.1^{b}$ & $1.08 \pm 0.05$ & $1.16 \pm 0.09$ & $-0.08 \pm 0.08$ & 0.09 \\
\hline $\mathrm{Cl}_{\text {paddick }}$ & b & $0.91 \pm 0.03$ & $0.86 \pm 0.04$ & $0.05 \pm 0.03$ & 0.03 \\
\hline
\end{tabular}

$\mathrm{V}_{\mathrm{x} \%}$ : volume receiving at least $\mathrm{x} \%$ of the dose. $\mathrm{D}_{\mathrm{x} \%}$ : dose received by at least $\mathrm{x} \%$ of the volume. $\mathrm{Cl}=$ conformity index

a: soft constraints, expected to be fulfilled in $70-80 \%$ of the patients according to EMBRACE II guidelines

b: not included as optimisation constraint

One of the potential limitations of the Halcyon system was identified in the field length with a single isocenter $(28 \mathrm{~cm})$. This study demonstrated that, also in the case of node-positive cervix patients, about $80 \%$ of the cases could be treated with one isocenter. The remaining cases were optimised with the automated double isocenter feature of the $\mathrm{H}$ system, which allows managing targets as long as $36 \mathrm{~cm}$ as positively demonstrated also by Kim [15]. Plans for more extended targets could be optimised with more manual procedures (requiring the use of sequential optimisations). Another potential limitation in the study derives from the fact that dose calculations were performed with $2.5 \mathrm{~mm}$ resolution for Halcyon and
$5 \mathrm{~mm}$ in the $\mathrm{x}-\mathrm{y}$ plane for Helical Tomotherapy. Although this might be a potential source of inconsistency in the comparison of the two datasets, we appraised its relevance on a subset of 5 patients recalculating the dose distributions in Halcyon with a $5 \mathrm{~mm}$ resolution. It resulted that the median percentage difference, computed over all the metrics used in the study, was $0.5 \%$ (the mean was $0.7 \%$ ) ranging from 0.0 to $4.1 \%$. The reason for this derives from the large volumes involved in the study for both targets and OARs, the fact that the voxel size in the HT calculations was $<0.1 \mathrm{~cm}^{3}$ and that no point metrics were used (consistently with ICRU recommendations [33]). In summary, we concluded that the 
spatial resolution might bias the results in principle but in a quite modest manner for the presently discussed data. Another limitation factor in the study is the choice of $5 \mathrm{~cm}$ as the field aperture which might be considered as a clinically unusual parameter setting (e.g. Hsieh [8] suggests the use of $2.5 \mathrm{~cm}$ ). The field size can affect the dose conformity and the OAR sparing, particularly in the regions where the PTVs shape changes rapidly in the longitudinal direction. The choice of $5 \mathrm{~cm}$ was according to standard clinical practice at the home institute. The most recent tomotherapy units are equipped with the option of dynamic jaw setting. In the present study, fixed jaw was used since clinically available at the institute. The potential (negative) impact of this choice is linked to a broader penumbra region in the cranio-caudal field edges. The use of dynamic jaw would therefore be advisable and might further improve the dose conformity and reduce the dose to some organs at risk (e.g. the bowels).

\section{Conclusion}

This retrospective treatment planning study, based on the dose constraints derived form the Embrace II study proto$\mathrm{col}$, suggested the fundamental equivalence between $\mathrm{Hal}-$ cyon based and Helical Tomotherapy based plans for the intensity-modulated rotational treatment of cervix uteri cancer. Different levels of sparing were observed for the bowels with $\mathrm{H}$ better protecting in the high-dose region and HT in the mid-low dose regions. The clinical impact of these differences should be further addressed.

\begin{abstract}
Abbreviations
CTV: Clinical target volume; CTV_E: Elective CTV; CTV_HR: High-risk CTV; CTV_LR: Low-risk CTV; CTV_N: Nodal CTV; EBRT: External beam radiation therapy; H: Halcyon; HT: Helical Tomotherapy; IG_IMRT: Image-guided Intensity Modulated Radiation Therapy; IMRT: Intensity Modulated Radiation Therapy; MLC: Multileaf collimator; PTV: Planning target volume; VMAT: Volumetric modulated arc therapy; N+: Node-positive.; N-: Nodenegative; CT: Computed tomography; MRI: Magnetic resonance images; GTV: Gross tumour volume; ITV: Internal target volume; ITV45: ITV for the primary tumour; PTV45: PTV for the primary tumour; PTV_N: PTV for the nodal volumes; SIB: Simultaneous integrated boost; OAR: Organ at risk; FFF: Flattening filter free; MU: Monitor units; SSD: Source surface distance; AAA: Anisotropic Analytical Algorithm; DVH: Dose-volume histograms; Cl: Conformity index
\end{abstract}

\section{Acknowledgements}

none.

\begin{abstract}
Authors' contributions
LC, SC, JPA, PK conceived the study. LC, SP, JW, AM, AF curated the treatment planning and the data collection. LC, AF performed the analysis. LC and JS drafted the manuscript. All authors reviewed and approved the manuscript.
\end{abstract}

\section{Funding}

none.

\section{Availability of data and materials}

The datasets used and analysed during the current study are available from the corresponding author.

\section{Ethics approval and consent to participate}

All procedures performed in studies involving human participants were in accordance with the ethical standards of the institutional and/or national research committee and with the 1964 Helsinki declaration and its later amendments or comparable ethical standards. The retrospective study has been approved by notification by the local ethics committee. Wherever necessary informed consent from the patients has been obtained.

\section{Consent for publication}

not applicable:

\section{Competing interests}

L. Cozzi acts as Scientific Advisor to Varian Medical Systems and is a Clinical Research Scientist at Humanitas Cancer Center. P. Kupelian is vice-president Medical Affairs at Varian Medical Systems. S. Chopra receives research funding from Varian not related to the current project. All other co-authors declare that they have no conflict interests.

\section{Author details}

1Department of Radiation Oncology, Advanced Centre for Treatment Research and Education in Cancer, Tata Memorial Centre, Homi Bhabha National Institute, Kharghar, Navi Mumbai, Maharashtra, India. ²Department of Radiation Oncology and Medical Physics, Tata Memorial Hospital, Tata Memorial Centre, Homi Bhabha National Institute, Parel, Mumbai, Maharashtra, India. ${ }^{3}$ Humanitas Research Hospital, Radiotherapy and Cancer Center Radiosurgery Dept, Via Manzoni 56, 20089 Milan-Rozzano, Italy. ${ }^{4}$ Varian Medical Systems, Palo Alto, CA, USA. ${ }^{5}$ Radiation Oncology Dept., University of California, Los Angeles, USA. ${ }^{6}$ Dept. of Biomedical Sciences, Humanitas University, Milan-Rozzano, Italy.

Received: 29 October 2019 Accepted: 19 January 2020

Published online: 30 January 2020

\section{References}

1. Isohashi F, Yoshioka Y, Mabuchi S, Konishi K, Koizumi M, Takahashi Y, et al. Dose-volume histogram predictors of chronic gastrointestinal complications after radical hysterectomy and postoperative concurrent nedaplatin based chemoradiation therapy for early-stage cervical cancer. Int J Radiat Oncol Biol Phys. 2013;85:728-34.

2. Chopra S, Dora T, Chinnachamy A, Thomas B, Kannan S, Engineer R, et al. Predictors of grade 3 or higher late bowel toxicity in patients undergoing pelvic radiation for cervical cancer: results from a prospective study. Int J Radiat Oncol Biol Phys. 2014;88:630-5.

3. Portelance L, Chao KS, Grigsby PW, Bennet H, Low D. Intensity-modulated radiation therapy (IMRT) reduces small bowel, rectum, and bladder doses in patients with cervical cancer receiving pelvic and Para-aortic irradiation. Int J Radiat Oncol Biol Phys. 2001;51:261-6.

4. Mundt AJ, Roeske JC, Lujan AE, Yamada S, Waggoner S, Fleming G, et al. Initial clinical experience with intensity-modulated whole-pelvis radiation therapy in women with gynecologic malignancies. Gynecol Oncol. 2001;82: 456-63.

5. Beriwal S, Gan GN, Heron DE, Selvaraj R, Kim H, Lalonde R, et al. Early clinical outcome with concurrent chemotherapy and extended-field, intensitymodulated radiotherapy for cervical cancer. Int J Radiat Oncol Biol Phys. 2007:68:166-71.

6. Cozzi L, Dinshaw KA, Shrivastava SK, Mahantshetty U, Engineer R, Deshpande DD, Jamema SV, Vanetti E, Clivio A, Nicolini G, Fogliata A. A treatment planning study comparing volumetric arc modulation with RapidArc and fixed field IMRT for cervix uteri radiotherapy. Radiother Oncol. 2008:89:180-91

7. Lian J, Mackenzie M, Joseph K, et al. Assessment of extended field radiotherapy for stage IIIC endometrial cancer using three-dimensional conformal radiotherapy, intensity-modulated radiotherapy and helical tomotherapy. Int J Radiat Oncol Biol Phys. 2008;70:935-43.

8. Hsieh $\mathrm{CH}$, Wie MC, Lee $\mathrm{H}-\mathrm{L}$, et al. Whole pelvic helical tomotherapy for locally advanced cervical cancer: technical implementation of IMRT with helical tomotherapy. Radiat Oncol. 2009;4:62.

9. Marnitz S, Koehler C, Burova E, Wlodarczyk W, Jahn U, Gruen A, et al. Helical tomotherapy with simultaneous integrated boost after laparoscopic staging in patients with cervical cancer: analysis of feasibility and early toxicity. Int J Radiat Oncol Biol. 2012;82:e137-43. 
10. Michels S, Poels K, Crijns W, Delombaerde L, De Roover R, Vanstraelen B, et al. Volumetric modulated arc therapy of head and neck cancer on a fast rotating O-ring linac: plan quality and delivery time comparison with a Carm linac. Radiother Oncol. 2018;128:479 [epub].

11. Flores-Martinez E, Kim G, Yashar C, Cervino L. Dosimetry study of the plan quality and dose to organs at risk on tangential breast treatments using the Halcyon linac. J Appl Clin Med Phys. 2019;20:7-58.

12. Riley C, Cox C, Graham S, Havran H, Kramer B, Netherton T, et al. Varian Halcyon dosimetry comparison for multiarc VMAT prostate and head and neck cancers. Med Dosim. 2018. https://doi.org/10.1016/j.meddos. 2018.06.004

13. Cozzi L, Fogliata A, Thompson S, Franzese C, Franceschini D, De Rose F, et al. Critical appraisal of the treatment planning performance of volumetric modulated arc therapy by means of a dual-layer stacked multileaf collimator for head and neck, breast and prostate. Tech Canc Res Treat. 2018;17:1-11.

14. Li T, Irmen P, Liu H, Shi W, Alonso-Basanta M, Zou W, Teo B, et al. Dosimetry performance and planning delivery efficiency of a dual-layer stacked and staggered MLC on treating multiple small targets: a planning study based on single isocenter multi-target stereotactic radiosurgery (SRS) to brain metastases. Front Oncol. 2019;9:7.

15. Kim M, Kennedy C, Scheuermann R, Freedman G, Li T. Whole breast and lymph node irradiation using Halcyon 2.0 utilizing automatic multi-isocenter treatment delivery and daily kilovoltage cone-beam computed tomography. Cureus. 2019:11:e4744.

16. Anamalayil S, Brady L, Grover S, Scheuermann R, Kennedy C, Mihailidis D, et al. Treatment of cervical cancer with a prototype flattening filter-free straight through linac with fast jawless MLC collimator. Int J Radiat Oncol Biol Phys. 2017;99:SE633.

17. Brady L, Scheuermann R, Anamalayil S, Kennedy C, Mihailidis D, Metz J. Robustness of extended field cervical target optimization techniques to isocenter offsets with a prototype fast jawless MLC system. Int J Radiat Oncol Biol Phys. 2017;99:SE642.

18. Mihailidis D, Brady L, Anamalayil S, Alonso-Basanta M, Scheuermann R, Kennedy C, Metz J. Rapid IMRT delivery for head and neck (H\&N) with a prototype jawless MLC system and a novel MV-CBCT panel. Int J Radiat Oncol Biol Phys. 2017:99:S230-1.

19. Kim H, Huq MS, Lalonde R, Houser CJ, Beriwal S, Heron DE. Early clinical experience with Varian Halcyon V2 linear accelerator: dual-isocenter IMRT planning and delivery with portal dosimetry for gynaecological cancer treatments. J Appl Clin Med Phys. 2019;20:111-20.

20. Li C, Chen J, Zhu J, Gong G, Tao C, Li Z, Lu J, Yin Y. Plan quality comparison for cervical carcinoma treated with Halcyon and trilogy intensity-modulated radiotherapy. J Cancer. 2019;10:6135-41.

21. Berger T, Seppenwoolde Y, Poetter R, Assenholt M, Lindegaard J, Nout R, de Leeuw A, et al. Importance of technique, target selection, contouring, dose prescription and dose planning in external beam radiation therapy for cervical cancer: evolution of practice from EMBRACE I to II. Int J Radia Oncol Biol Phys. 2019;104:885-94.

22. Lim T, Dragojevic I, Hoffman D, Flores-Martinez E. Characterization of the Halcyon multileaf collimator system. J Appl Clin Med Phys. 2019;20:106-14.

23. De Roover R, Crijns W, Poels K, Michiels S, Nulens A, Vanstraelen B, et al. Validation and IMRTNMAt delivery quality of a pre-configured fast-rotating O-ring linac system. Med Phys. 2019;46:328-39.

24. Lloyd S, Lim T, Fave X, Flores-Martinez E, Atwood T, Moiseenko V. TG-51 reference dosimetry for the Halcyon: a clinical experience. J Appl Clin Med Phys. 2018;19:98-102.

25. Ulmer W, Harder D. A triple gaussian pencil beam model for photon beam treatment planning. Z Med Phys. 1995;5:25-30.

26. Mackie T, Holmes T, Swerdloff S, Reckwerdt P, Deasy J, Yang J, et al. Tomotherapy: a new concept for the delivery of dynamic conformal radiotherapy. Med Phys. 1993;20:1709-19.

27. Fenwick J, Tome W, Soisson E, Mehta M, Mackie R. Tomotherapy and other innovative IMRT systems. Sem Rad Oncol. 2006;16:199-208.

28. Grigorov G, Kron T, Wong E, Chen J, Sollazzo J, Rodrigues G. Optimisation of helica tomotherapy treatment plans for prostate cancer. Phys Med Biol. 2003;48:1933-43.

29. Jeraj R, Mackie T, Balog J, Olivera G, Pearson D, Kapatoes J, et al. Radiation characteristics of helical tomotherapy. Med Phys. 2004;31:396-404.

30. Paddick I. A simple scoring ratio to index the conformity of radiosurgical treatment plans. J Neurosurg. 2000;93:219-22.
31. De Roover R, Crijns W, Poels K, Michels S, Nulens A, Vanstraelen B, et al. Validation and IMRTNMAT delivery quality of a preconfigured fast-rotating O-ring linac system. Med Phys. 2019;46:328-39.

32. Ezzel G, Burmeister J, Dogan N, et al. IMRT commissioning: multiple institute planning and dosimetry comparisons, a report from AAPM task group 119. Med Phys. 2009;36:5359-73.

33. ICRU Report 83. Prescribing recording and reporting photon beam intensity-modulated radiation therapy (IMRT). ICRU Reports, Volume 10 number 1.2010.

\section{Publisher's Note}

Springer Nature remains neutral with regard to jurisdictional claims in published maps and institutional affiliations.
Ready to submit your research? Choose BMC and benefit from:

- fast, convenient online submission

- thorough peer review by experienced researchers in your field

- rapid publication on acceptance

- support for research data, including large and complex data types

- gold Open Access which fosters wider collaboration and increased citations

- maximum visibility for your research: over $100 \mathrm{M}$ website views per year

At BMC, research is always in progress.

Learn more biomedcentral.com/submissions 